\title{
Parabolized stability equation models for predicting large-scale mixing noise of turbulent round jets
}

\author{
D. Rodríguez ${ }^{1 *}$, A. Samanta ${ }^{1 \dagger}$, A. V. G. Cavalieri ${ }^{2 \ddagger}$, \\ T. Colonius ${ }^{1 \S}$ and P. Jordan ${ }^{2 \uparrow}$ \\ ${ }^{1}$ Department of Mechanical Engineering, California Institute of Technology, Pasadena, USA \\ ${ }^{2}$ Institute Pprime, Poitiers, France
}

\begin{abstract}
Parabolized stability equation (PSE) models are being developed to predict the evolution of low-frequency, large-scale wavepacket structures and their radiated sound in highspeed turbulent round jets. Linear PSE wavepacket models were previously shown to be in reasonably good agreement with the amplitude envelope and phase measured using a microphone array placed just outside the jet shear layer. ${ }^{1,2}$ Here we show they also in very good agreement with hot-wire measurements at the jet centerline in the potential core, for a different set of experiments. ${ }^{3}$ When used as a model source for acoustic analogy, the predicted far field noise radiation is in reasonably good agreement with microphone measurements for aft angles where contributions from large-scale structures dominate the acoustic field. Nonlinear PSE is then employed in order to determine the relative importance of the mode interactions on the wavepackets. A series of nonlinear computations with randomized initial conditions are use in order to obtain bounds for the evolution of the modes in the natural turbulent jet flow. It was found that nonlinearity has a very limited impact on the evolution of the wavepackets for $S t \geq 0.3$. Finally, the nonlinear mechanism for the generation of a low-frequency mode as the difference-frequency mode ${ }^{4,5}$ of two forced frequencies is investigated in the scope of the high Reynolds number jets considered in this paper.
\end{abstract}

\section{Introduction}

Despite advances in large eddy simulations, accurate and fast prediction of the aerodynamic noise emitted from high speed jets remain challenging and computationally expensive. On the other hand, numerical jet noise predictions based on the acoustic analogy ${ }^{6}$ approach require an universal definition of the statistical sound sources, which is presently lacking. In this paper a different approach is pursued, based on a coarse description of the large-scale turbulent structures and a subsequent projection of the pressure fluctuations associated with these structures to the far field.

The large-scale structures are modeled as instability wavepackets of the mean turbulent flow. Their relatively large scale make the evolution of the wavepackets relatively insensitive to the finer-scale turbulence, except for their impact on the evolution of the jet mean flow field. This particular approach has been employed for some decades ${ }^{7-10}$ in the study of forced supersonic jets, for which the measured near field fluctuations were found to be in good agreement with predictions of linear stability theory. ${ }^{11}$

In the case of natural (unforced) jets of interest here, this approach has only recently begun to deliver satisfactory quantitative predictions. Suzuki and Colonius ${ }^{12}$ showed that pressure fluctuations measured just outside the jet shear layer were consistent with the corresponding evolution as inferred from modeling them as linear instability waves, computed using locally parallel flow analysis of the jet mean flow field. The

\footnotetext{
*Postdoctoral Scholar, AIAA Member

$\dagger$ Postdoctoral Scholar, AIAA Member; currently Assistant Professor, Indian Institute of Science

${ }^{\ddagger} \mathrm{Ph}$. D. Student

$\S$ Professor, AIAA Associate Fellow

I Research Scientist
} 
slowly diverging nature of the mean flow suggests that more sophisticated techniques should be considered for the computation of the wavepackets. In Gudmundsson and Colonius ${ }^{13}$ and in this paper, Parabolized Stability Equations (PSE) are used in order to take into account the mild variation of the jet mean flow along the axial direction. Another remarkable advantage over classic linear stability theory is that nonlinear interactions between different frequencies and azimuthal wavenumbers can be considered in the computation of the corresponding wavepackets. This approach has been already employed in the literature both in its linear ${ }^{13,14}$ and nonlinear ${ }^{15}$ versions, with promising results. Alternatively, global instability analysis could also be used to provide the description of the wavepackets, ${ }^{16}$ but at a much higher computational expense.

In our past studies ${ }^{12}$, the microphone arrays were carefully positioned in an attempt to place them in a region close to the shear layer where the measured signal is predominantly hydrodynamic in nature. In practice, depending upon specific conditions, noise from uncorrelated acoustic sources are also picked up by these microphones, which if removed can provide even better match with the PSE predictions. This filtering may be achieved via a Proper Orthogonal Decomposition (POD) that separates signals into uncorrelated components. The results presented in Gudmundsson ${ }^{1}$ and Gudmundsson and Colonius ${ }^{2}$ demonstrate a remarkable agreement of the linear PSE evolution with the most energetic POD mode, especially for the lower order modes and even beyond the potential core.

Evidence suggests that the wave-packet structures that dominate the near pressure field are sufficiently acoustically efficient so as to also represent a significant portion of the far-field sound. ${ }^{17}$ In Colonius et al. ${ }^{18}$ this possibility was analyzed in conjunction with the acoustic projection method of Reba et al. ${ }^{17}$ where wavepackets, directly measured along the near-field array were projected to the acoustic far field using a Kirchhoff surface methodology. The far acoustic field at aft angles was reasonably well predicted by the projected linear PSE results within a few $\mathrm{dB}$ of the measurements.

The first part of the present contribution revisits our linear PSE methods including the far-field projection, albeit with a different set of data. The present hot-wire measurements, obtained in a series of experiments at the Institute Pprime in Poitiers are compared with linear PSE predictions for low $\left(M_{j}=0.4\right.$ to 0.6) Mach number jets, which are then used to scale the otherwise arbitrary amplitude of the linear wavepackets. Following Crow ${ }^{19}$ and Crighton ${ }^{20}$, the PSE results are then used to build a model source for the Lighthill's acoustic analogy ${ }^{6}$. Comparison between the sound pressure levels at the far field predicted by this methodology and acoustic measurements show reasonably good agreement. The experimental results are presented in a companion paper in the same conference ${ }^{3}$.

The second part of the paper deals with the application of nonlinear PSE to unforced turbulent jets. As opposed to linear models, nonlinear computations are sensitive to the definition of the initial conditions for the modes, as well as to the choice of the frequency and azimuthal modes retained in the simulations. Besides these formulation-related problems, the selection of meaningful initial conditions requires further investigation. The nature of unforced turbulent jet flows is such that the fluctuations in the near nozzle region are not adequately described in a deterministic manner. While high-fidelity computations or measurements can be used to obtain accurate time series to be used as initial conditions, they would only be representative of one possible realization. On the other hand,using spectral averaging techniques thet might provide a smooth ("universal") disturbance spectrum at the nozzle exit does not provide the phase difference between modes that gives rise to differing nonlinear interactions. An intermediate method is used here, in which the near field pressure measurements of Suzuki and Colonius ${ }^{12}$ are used in order to compute averaged initial conditions. A series of computations are then performed in which both the amplitudes and phases of these initial conditions are randomized, in order to determine bounds for the nonlinear evolution of the wavepackets.

The paper is organized as follows. § II presents the nonlinear PSE equations and discusses the algorithm used in the numerical solution. § III compares linear PSE computations with hot-wire measurements, and compares the results of using the linear wavepackets as input for the acoustic analogy with acoustic measurements at the far field. The investigations conducted using nonlinear PSE are described in $\S$ IV.

\section{Parabolized Stability Equations}

Linear and nonlinear PSE are employed here in order to model the spatial evolution of the wavepackets. PSE $^{21-23}$ represent a generalization of the parallel-flow linear stability theory (LST) for flows with a mild variation on the streamwise direction, also permitting the nonlinear interaction of the different modes. The total flow field $q$ is decomposed into a mean (time-averaged) and axisymmetric component $\bar{q}$ and its 
fluctuations:

$$
q=\bar{q}+q^{\prime}
$$

where $q=\left[u_{x}, u_{r}, u_{\theta}, T, \rho\right]^{T}$ is the vector of fluid variables. Only round-nozzle jets are considered in this work, and consequently the mean flow is assumed to be axisymmetric; the fluctuating part is then written as a sum of Fourier modes in the azimuthal direction and in frequency where $m$ is the azimuthal wavenumber, $\omega$ is the fundamental circular frequency, and $\chi_{m n}$ is the modal function corresponding to the mode $(m, n)$. In the practical solution of nonlinear PSE, the azimuthal and frequency modes must be truncated, involving a finite number $(M, N)$ of modes. The unresolved modes are then formally gathered in the term $q^{\prime \prime}$, and the fluctuation field is written as

$$
q^{\prime}(x, r, \theta, t)=\sum_{n=-N}^{N} \sum_{m=-M}^{M} \chi_{m n}(x, r) \exp (\mathrm{i}(m \theta-n \omega t))+q^{\prime \prime}
$$

The mean flow is a function of the axial and radial directions $(x, r)$, but a slow variation of its properties along the axial direction is assumed. This assumption permits the decomposition of $\chi_{m n}$ into a slowly varying shape function (that evolves in the same scale as the mean flow) and a rapidly varying wave-like part:

$$
\chi_{m n}(x, r)=A_{m n}(x) \cdot \hat{q}_{m n}(x, r)=\exp \left(\mathrm{i} \int_{x} \alpha_{m n}(\xi) d \xi\right) \cdot \hat{q}_{m n}(x, r) .
$$

Here $\alpha_{m n}(x)$ is a complex axial wavenumber, for which a mild variation is also assumed. It is important to stress that the separation in scales between the mean flow, and the modal shape functions on one hand, and the modal wavelengths associated with $\alpha_{m n}$ on the other is a necessary hypothesis in the derivation of the PSE. However, for low frequencies the wavelength can be comparable to the extent of the potential core. This issue will be discussed further in the following sections.

Introducing the decomposition (2-3) into the compressible Navier-Stokes, continuity and energy equations and substracting the terms corresponding to the mean flow, we arrive at the system of equations

$$
\left[\mathbf{A}(\bar{q}, \alpha, m, n \omega)+\mathbf{B}(\bar{q})+\mathbf{C}(\bar{q}) \frac{\partial}{\partial x}+\mathbf{D}(\bar{q}) \frac{\partial}{\partial r}+\frac{1}{R e} \mathbf{E}\right] \hat{q}=\frac{\hat{F}_{m n}}{A_{m n}}+\frac{F_{m n}^{\prime \prime}}{A_{m n}} .
$$

The linear operators $\mathbf{A}$ to $\mathbf{E}$ can be found in Gudmundsson ${ }^{1}$. For brevity, the subscript have been dropped from the shape function and wavenumber in the previous expression. The left-hand-side on (4) is a linear spatial operator for the mode $(m, n)$, while the right-hand-side accounts for all the nonlinear contributions to the modal evolution. Linear PSE are obtained setting the right-hand-side equal to zero. The contributions resulting from nonlinear interactions of the resolved modes are comprised in the function $\hat{F}_{m n}(x, r)$, while those interactions in which the unresolved modes are involved are formally gathered in the function $F_{m n}^{\prime \prime}$. In nonlinear computations considering laminar or transitional flows all the dynamically relevant modes are resolved, and $F_{m n}^{\prime \prime}$ is neglected. In the case of the natural turbulent jets of interest here, a broad spectrum exists and the truncation of the modes is always arbitrary. Consequently, the function $F_{m n}^{\prime \prime}$ is not necessarily negligible and needs to be modeled. This issue is discussed further in $\S I I$.B.

Following from the slow axial variation assumed for $\hat{q}$, the second axial derivatives on the viscous terms are neglected, so that the system of equations can be integrated along the $x$-direction. The decomposition of (3) is ambiguous in that the evolution of $\chi$ can be absorbed into either the shape function $\hat{q}_{m n}$ or the complex amplitude $A_{m n}$ corresponding to the wave-like behavior. Following Herbert, ${ }^{23}$ the normalization condition

$$
\int_{0}^{\infty} \hat{q}^{\dagger} \frac{\partial \hat{q}}{\partial x} r d r=0
$$

is imposed individually to every mode, removing the exponential dependence on the shape function $\hat{q}$. Here the superscript $\dagger$ denotes complex conjugation.

The system of equations (4) is discretized using fourth-order central finite differences in the radial direction, closing the domain with the characteristic boundary conditions of Thompson. ${ }^{24}$ The streamwise derivative is approximated using an implicit first-order Euler scheme with a relatively large step size $\Delta x$, 
in order to prevent the appearance of instabilities in the marching procedure, related to ellipticity issues. ${ }^{25}$ For each successive axial location, the system (4) is solved iteratively: the $\alpha$ and $\hat{q}$ converged in the previous location are used as initial guess. The nonlinear forcing term is computed in physical space, and then transformed back to Fourier space. ${ }^{15,26}$ Then, a new approximation to $\hat{q}$ is obtained from solving (4). Finally, the wavenumber $\alpha$ is updated on each step using the normalization condition (5). The iteration continues until the correction computed for all $\alpha_{m n}$ is below a certain threshold, taken as $10^{-8}$ in the present computations.

\section{A. Initial conditions}

In line with the parabolic solution procedure for the PSE, adequate initial conditions must be provided for the shape functions $\hat{q}_{m n}\left(x=x_{0}, r\right)$ and the wavenumbers $\alpha_{m n}\left(x=x_{0}\right)$. These initial conditions are obtained from the solution of the linear stability problem for the velocity profile at the initial axial location $x_{0}$, which is typically chosen just downstream of the nozzle lip. In all cases, the LST eigenmode corresponding to the shear-layer instability is imposed to every mode.

In the case of linear computations, the evolution of each mode does not depend on the initial amplitude or phase. Consequently, the linear PSE can be solved with any arbitrary initial amplitude $A_{m n}\left(x=x_{0}\right)$, and then rescaled to simulation data or experimental measurements. Data from two different experimental settings is considered in the present paper, as will be discussed below. In both cases, time series of velocity or pressure are measured at a limited number of locations along the axial direction. Amplitude distributions for each mode are extracted from these data, and used to determine the values $A_{m n}\left(x=x_{0}\right)$ that result in a better least-square fit between the PSE wavepackets and the experiments. In some cases, phase information between the different experimental probes is retained, and more sophisticated techniques like Proper Orthogonal Decomposition (POD) can be introduced in order to filter out uncorrelated contributions to the total signal, providing a clearer description of the physical wavepacket.

In the case of nonlinear computations, the evolution of the different modes is coupled and depends on their relative amplitudes and phases. A critical aspect of the nonlinear computations is the requirement to provide meaningful initial conditions to the modes in the near-nozzle region. Experimental data or high-fidelity simulations can be used in order to determine these initial amplitudes and phases, but their physical interpretation is not straight forward. In the case of the natural (unforced) jets of interest here, the disturbance conditions at the nozzle lip are not imposed, but experience continuous changes due to many uncertainty sources in the natural turbulent jet. In the present paper, the non-deterministic nature of the initial conditions is dealt with by assuming some degree of universality in the disturbance spectra. The experimental data is used to extract mean modal amplitudes for the different eigenmodes. Then, the initial amplitudes and phases are varied randomly in a series of simulations in order to determine bounds for the predicted nonlinear wavepackets. More details on the nonlinear PSE simulations will be given in $\S I V$.

\section{B. Mean flow distortion and turbulence modeling}

The non-linear PSE approach was originally devised for investigating laminar and transitional flows ${ }^{23}$. In these cases, a similarity solution like the Blasius solution for flat-plate boundary layer or the homogeneous mixing layer is prescribed as the mean flow. Then, the nonlinear interaction of the instability waves produces zero-frequency modes $\chi_{m 0}$, representing a distortion of the mean flow. This interaction is responsible for the saturation of the modes in transitional flows. However, this interpretation of the mean flow distortion is not adequate for natural turbulent jets, where a broad range of scales is present from the nozzle lip.

In the scope of this paper, non-linear PSE constitute a model for the evolution of the wavepackets along the axial direction. Only the coherent large-scale turbulent structures are then accounted for in the equations explicitly, while the incoherent part of the turbulent motion needs to be modeled. This decomposition of the fluctuating parts in the flow is in line with the requirement of truncating the number of azimuthal and frequency modes in the non-linear PSE; invariably, a range of resolved and unresolved scales is present in the computations, as was shown in equation (2) where $q^{\prime \prime}$ stands for the unresolved part of the fluctuations. The interaction between the random fluctuations and the wavepackets has partially been introduced by using the true turbulent mean flow as the base flow for the PSE computations. In this sense, it can be theorized that a separation in temporal and spatial scales exists between the large scales and random turbulence, so that the evolution of the former is governed by the mean flow and only has an indirect effect of the latter through its impact on the mean flow. Consequently, the original mean flow used in the PSE computations should be kept unaltered. 
A step further in the model can be achieved if the nonlinear interactions between the wave-packets (the resolved modes) and the uncoherent turbulence (or unresolved modes), appearing as the function $F_{m n}^{\prime \prime}$ in equation (4) are modeled through permitting the mean flow distortion to develop. In this case, the mean flow distortion generated non-linearly represents the departure of the mean flow within the PSE computations, caused by the truncation of the higher-order modes. This approach is very similar to the shift mode used in the Reduced Order Models proposed by Noack and co-workers ${ }^{27}$.

Implicit in the idea that the small scales act on the mean flow through the Reynolds stresses increasing the spread rate of the mean jet, is the consideration that the effective viscosity seen by the mean flow is substantially higher than the molecular viscosity. Following classic derivations for mean turbulent flows ${ }^{28}$ , an axial distribution of the effective eddy viscosity can be obtained from the spread of the shear-layer, and introduced in the PSE equations for the mean flow distortion. The effect of the unresolved fluctuations $q^{\prime \prime}$ on the resolved wavepackets is introduced via the linear interaction of the modes with the mean flow $\bar{q}$, and also via the nonlinear interaction with the mean flow distortion modes $\chi_{m 0}$, that act as an energy sink. However, in exploratory tests it was found that introducing the eddy viscosity model for the mean flow distortion modes did not alter in a significative manner the evolution of the wavepackets. Finally, the eddy viscosity model was not used in the nonlinear computations presented in $§ \mathrm{IV}$.

\section{Linear PSE models and their acoustic far field}

\section{A. Comparison with phased microphone array}

This section summarizes the results obtained in previous works ${ }^{1,2}$ regarding the application of the linear PSE approach to modeling the wavepackets, in conjunction with using a Kirchhoff surface method to project the pressure disturbances to the far-field. ${ }^{18}$

The data obtained in a series of experiments conducted at the SHJAR facility at NASA Glenn Research Center was employed. A number of different experimental conditions, defined in Tanna's case matrix, ${ }^{29}$ were set. Mean flows were measured using stereoscopic PIV ${ }^{30}$ and used as the base flow in the linear PSE computations. In addition, near-field pressure fluctuations were measured using a phased microphone array. The microphone array was placed just outside the jet shear layer, in the region dominated by linear hydrodynamic disturbances and consequently best suited to recover the pressure imprint of the wavepackets with a minimum of acoustic contamination. ${ }^{12}$ The pressure time series were Fourier transformed in time and in the azimuthal direction, retaining the phase information, and then used to scale the amplitude of the wavepackets delivered by linear PSE computations. The experimental and predicted pressure amplitude envelopes and phases for a cold $M_{j}=0.9$ jet are shown in figure 1 . The experimental signal contains some contamination of the amplitude not caused by the wavepackets, that manifests itself in the disagreement with the linear PSE results downstream of the disturbance pressure peak. Proper Orthogonal Decomposition was used in order to filter out the signal contributions that were uncorrelated along the microphone array, resulting into excellent agreement with linear PSE predictions for all except the lower frequencies considered (square symbols in figure 1).

The acoustic projection method of Reba et al. ${ }^{17}$ was employed then in order to determine the noise radiated to the far-field by the computed wavepackets. From the PSE results, the fluctuation pressure was measured along the near-field array and then projected using a Kirchhoff surface methodology. ${ }^{18}$ Left part of figure 2 shows the pressure amplitudes at the array location, extracted from the PSE results and scaled with the microphone data. Right part of figure 2 compares the far-field sound pressure levels predicted by the acoustic projection method with experimental measurements. Here the angle with the axis is measured with respect to the forward direction. In spite of the apparent mismatch between the near-field PSE results with microphone data downstream of the array, the far acoustic field at aft angles is reasonably well predicted, within a few $\mathrm{dB}$ of the measurements. However, it was shown that the use of the Kirchhoff surface method in combination with the PSE wavepackets did not deliver consistent results when the Mach numbers were low. Besides the fact that the efficiency of the noise radiation mechanism is highly dependent on the Mach number, numerical inaccuracies on the pressure field associated with the PSE solution are deemed responsible for the disagreement. The pressure amplitude in the PSE modes decay in the radial direction approximately

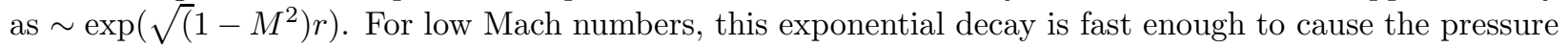
signal extracted at the array location to be contaminated with numerical noise. Consequently, projecting these pressure distributions to the far-field produces unphysical results in most cases. 

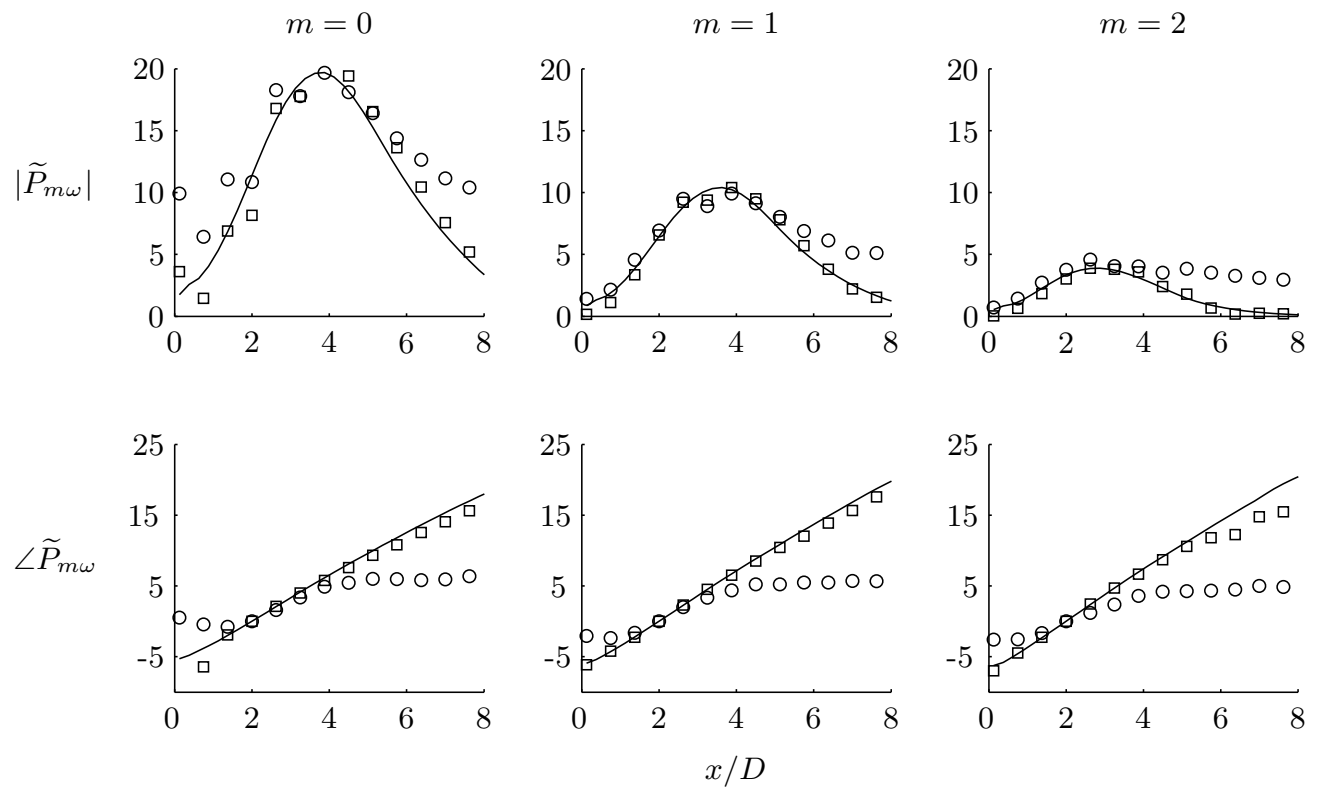

Figure 1. Pressure amplitude and phase-angle along the microphone array for the cold $M_{j}=0.9$ jet: $\circ$, measurements; $\square$, first POD-mode; - , PSE, at frequency $S t=0.35$. Extracted from Ref. 2.
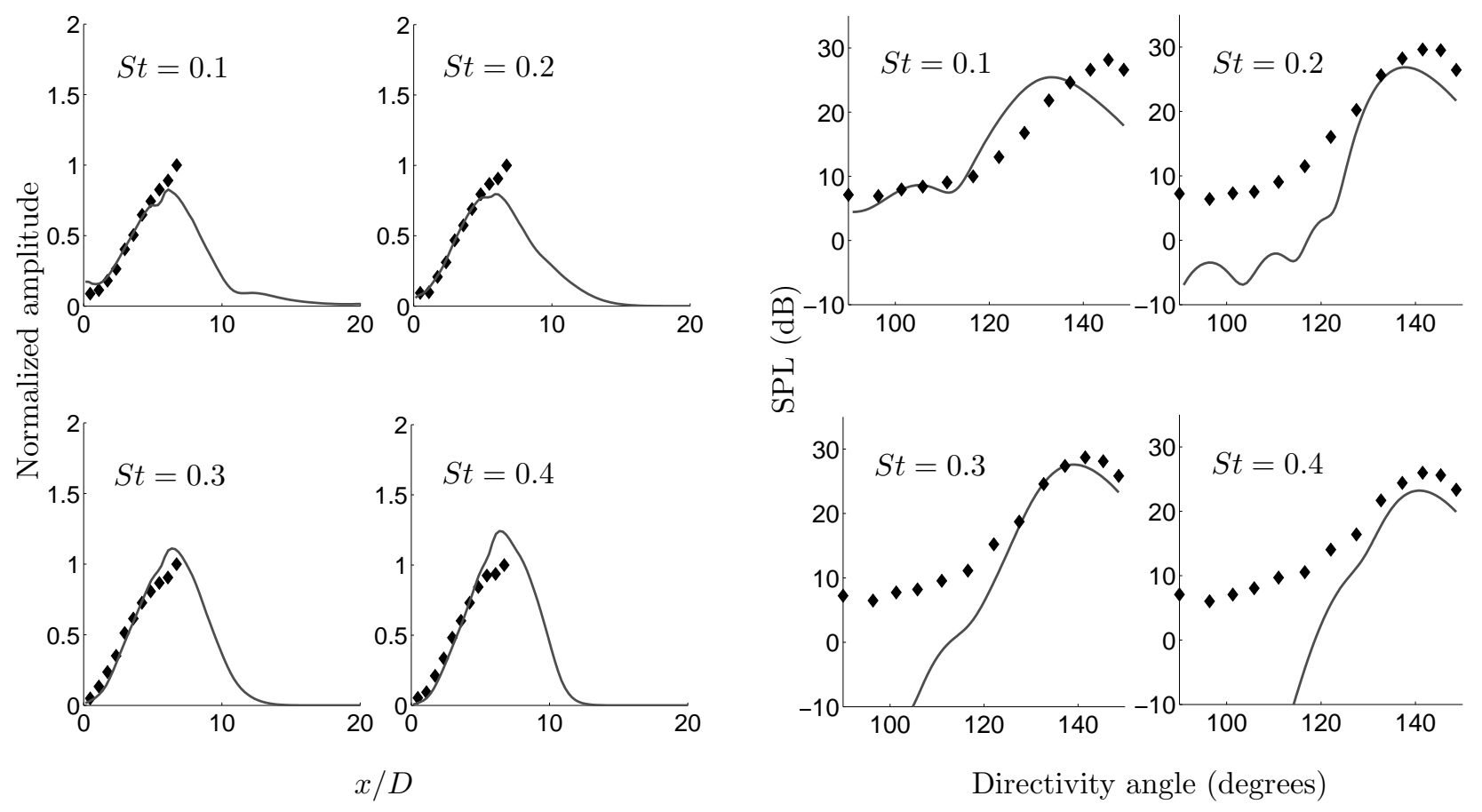

Figure 2. Left: Pressure correlation along microphone array. Right: Far-field projection. ( $\downarrow)$ near-field and far-field microphone data, respectively; (-) PSE solution. For $M_{j}=0.9, T_{j} / T_{\infty}=2.7, m=0$. Extracted from Ref. 18.

\section{B. Comparison with Poitiers' hot-wire measurements}

In this section the wave-packet evolution predicted by linear PSE is compared with hot-wire measurements obtained for cold subsonic jets. The set of experiments were carried out in the Bruit et Vent anechoic 


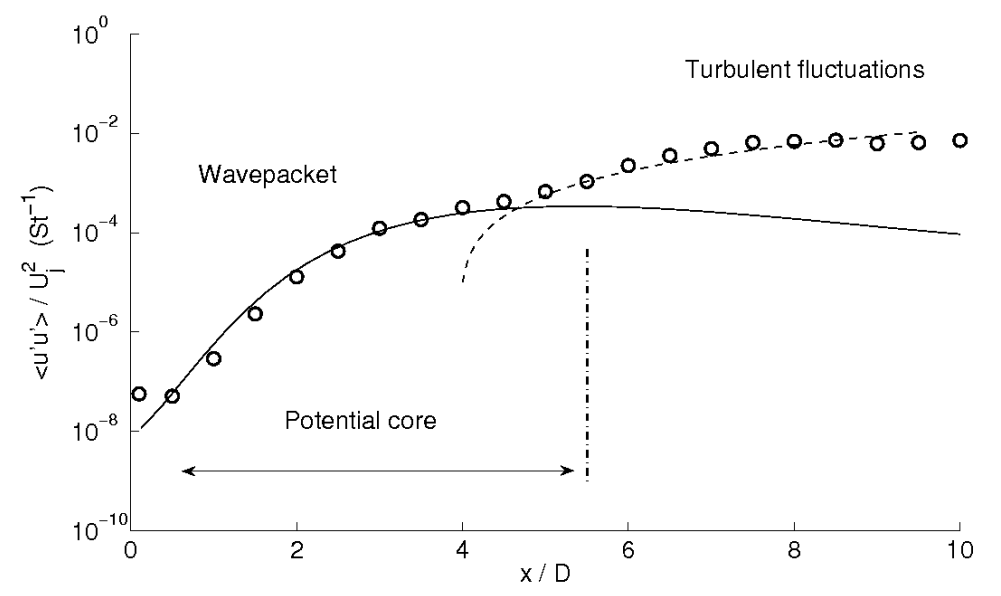

Figure 3. Different regions on the jet centerline hot-wire measurements. The curves represent a notional separation between the large-scale velocity fluctuations associated with the centerline behavior of wavepackets and finer-scale turbulence in the merging shear-layers.

facility at the Centre d'Etudes Aérodinamiques et Thermiques (CEAT), at the Institut Pprime in Poitiers, France. More details on the experimental setup can be found elsewhere. ${ }^{3,31}$ Three different Mach numbers $\left(M_{j}=0.4,0.5,0.6\right)$ are considered here. The Reynolds number based on the nozzle diameter and jet velocity was $O\left(10^{5}\right)$, and a boundary layer trip was used to force transition upstream of the nozzle exit. Hot-wire measurements were used in order to determine the mean flow used in the PSE computations. In addition, time-series of hot-wire measurements were obtained at the jet centerline, and used to scale the linear PSE results.

For the present experimental setup, the fine-scale turbulent fluctuations present in the nozzle boundary layer are absent inside of the jet potential core, where the flow is uniform and laminar. Consequently, the velocity fluctuations measured at the jet centerline must correspond solely to the imprint of the instability wavepackets, whose spatial structure extends in the radial direction well beyond the shear-layer. In addition, the symmetry properties of $m \neq 0$ modes imply that only axisymmetric modes can have a non-vanishing axial velocity at the axis. The result is that hot-wire measurements in the jet centerline can be compared directly to the axisymmetric modes computed using PSE, along the potential core. Towards and downstream of the end of the potential core, the shear-layer has spread so that a wide range of turbulent fluctuations are present at the centerline, overwhelming the contribution of the wavepackets to the hot-wire signal. Figure 3 illustrates the two distinct regions in the hot-wire velocity distributions.

Figure 4 compares the axial velocity component at the centerline predicted by linear PSE for different frequencies $S t$ with the hot-wire measurements. Very small quantitative differences appear for the Mach numbers explored. The PSE results have been arbitrarily scaled in order to match the experimental amplitudes in the fifth station. Except for the lower frequencies $S t=0.1-0.2$, very good agreement exists in the amplitude envelope in a region extending from the nozzle lip until approximately 5 diameters downstream, coinciding with the end of the mean potential core, for the reasons explained above. This observation is a further evidence that coherent, large-scale turbulent structures are also present in natural turbulent jets, and their evolution can be modeled using wavepackets, at least in the region between the nozzle and the end of the potential core.

In addition to the mismatch between wavepackets and measurements downstream the potential core closure, a second point of disagreement in figure 4 appears for the lower frequencies. While rather speculative, it can be argued that the wavelengths associated with these low frequencies are of the same order of magnitude of the nozzle diameter and the potential core. The separation of scales assumed in the derivation of the PSE approach is weak in these cases, and discrepancies are to be expected.

Figure 4 also shows that the both the wavepacket and measured amplitudes are decreased as the jet Mach number $U_{j}$ is increased, while the shape functions are nearly identical for the different Mach numbers (see figure 5). This is a known result in linear stability theory, that predicts that for subsonic flow, compressibility reduces the growth rates of inflectional instability waves. This issue is discussed further elsewhere. ${ }^{3}$ The 

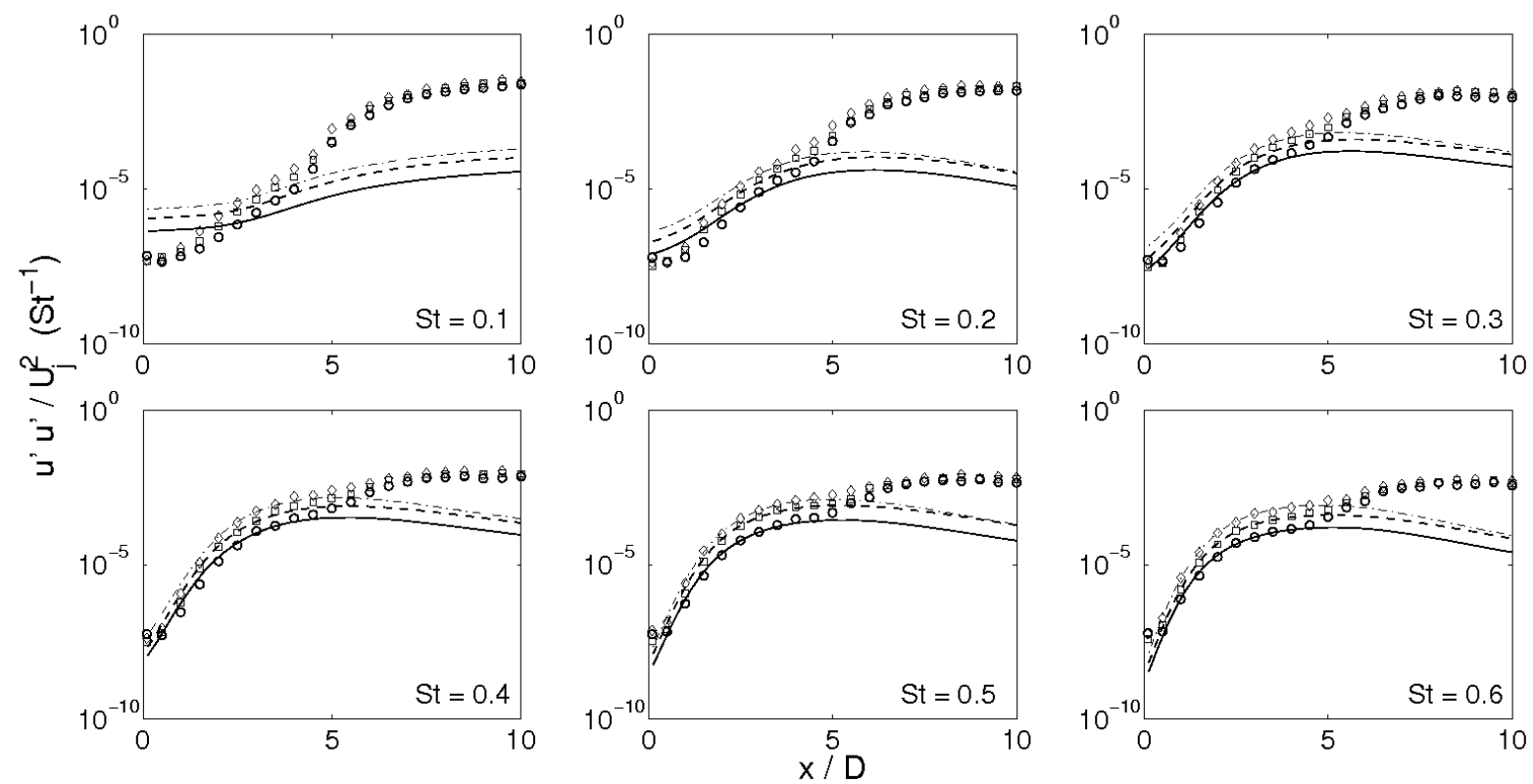

Figure 4. Comparison of the centerline velocity predicted by linear PSE (lines) with experimental measurements (symbols), for $M_{j}=0.4$ (dot-dashed lines, diamonds), $M_{j}=0.5$ (dashed lines, square) and $M_{j}=0.6$ (solid lines, circles)

real part of the axial velocity component of the shape functions, $\chi^{u}(x, r)$, for three frequencies and $M_{j}=0.4$ and $M=0.6$, are shown in the figure 5 . In the vicinity of the nozzle, the structure of the wavepackets is centered in the shear-layer $(r / D=0.5)$. As the waves evolve downstream, the wavepacket structure spreads with the mean flow and the oscillations inside the potential core become of the same order than those on the shear-layer.

\section{Far-field projection}

It has been shown that linear PSE deliver wavepackets that are in good agreement with experimental measurements of large-scale structures inside the turbulent jet. These large-scale structures are known to be responsible for the super-directive noise radiation, peaking in the aft direction. As stated in the introduction, the main objective of the present investigation is to predict the far field noise radiation originated by the wavepackets. In Reba et al. ${ }^{17}$ and Colonius et al. ${ }^{18}$ the disturbance pressure of the wavepackets was projected to the far field by means of using a Kirchhoff surface methodology. As was mentioned in §IIIA, the combination of PSE-obtained wavepackets and Kirchhoff surface method does not deliver good results for low Mach numbers. A different methodology is employed here, based on the acoustic analogy ${ }^{6}$, that makes used of the velocity distribution at the jet centerline, instead of the pressure at the near-field. Crow ${ }^{19}$ $\left(\right.$ see also Crighton ${ }^{20}$ ) proposed a wavepacket model based on an axially-coherent line distribution of aligned quadrupoles. We modify this model in order to use the measured mean velocity and the PSE modes to form the source term

$$
T_{11}(x, \omega)=4 \pi \rho_{0} \delta(r) \int_{0}^{\infty} \bar{u}(x, r) u(x, r, \omega) r \mathrm{~d} r
$$

where $\rho_{0}$ is the density in the far field, and $u(x, r, \omega)=\chi^{u}(x, r)$ is the axial velocity fluctuation taken from the PSE modes. The radial integration is justified for the present Mach and Strouhal numbers, by an assumption of radial compactness. ${ }^{32}$ With this model source, the acoustic field is obtained by

$$
p(\xi, t)=\frac{1}{4 \pi} \iiint\left[\frac{1}{|\xi-\mathbf{x}|} \frac{\partial^{2} T_{11}}{\partial x^{2}}(x, \omega) \mathrm{e}^{\mathrm{i} \omega \tau}\right]_{\tau=t-\frac{|\xi-\mathbf{x}|}{c}} \mathrm{~d} \mathbf{x} .
$$


$M_{j}=0.4$
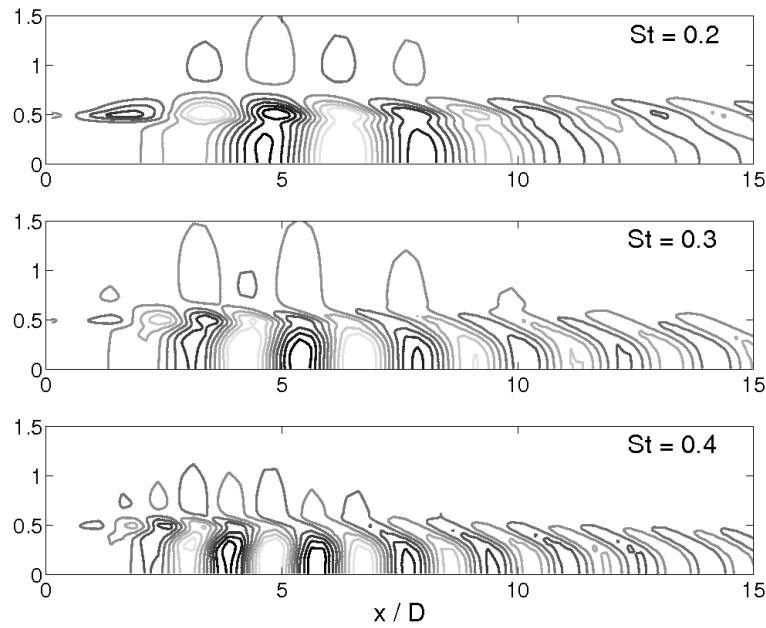

$M_{j}=0.6$
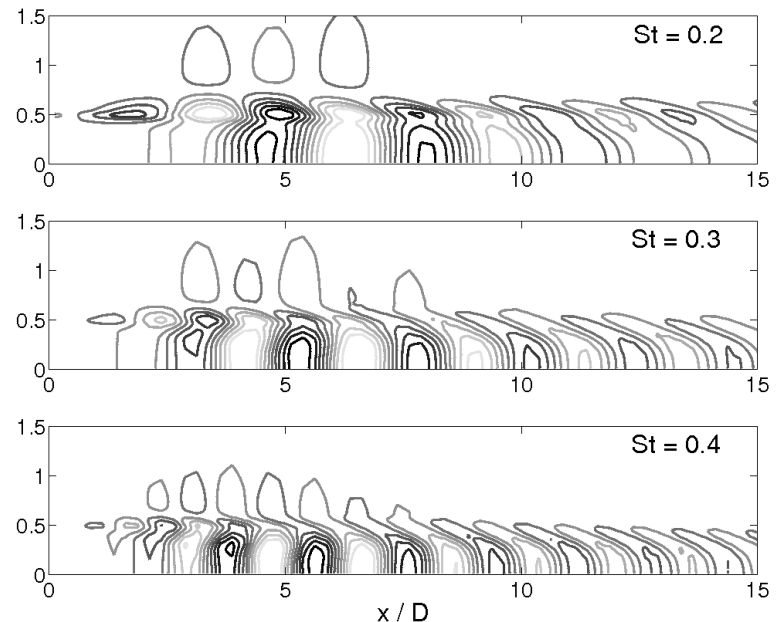

Figure 5. Axial velocity perturbation $\chi^{u}(x, r)$ predicted by linear PSE, for $M_{j}=0.4$ and $M_{j}=0.6$

The coordinates $\xi$ in equation (7) correspond to the projected far field. To compute the unbounded integral, the radially integrated source is fit to a simpler analytical function whose amplitude is given by $A(x)=C \mathrm{e}^{-\left(x_{1}-x_{c}\right)^{2} /\left(L+a x_{1}\right)^{2}}$, and whose phase speed is taken to be constant. The sample fits shown in figure 6 demonstrate the efficiency of this approach for the current PSE data. We note that the Gaussian function is similar to that used in Reba et al. ${ }^{17}$ and Koenig, ${ }^{33}$ but in the present case we are not using it as a model, but simply as a device to accurately compute the integral given the PSE source term.

(a)
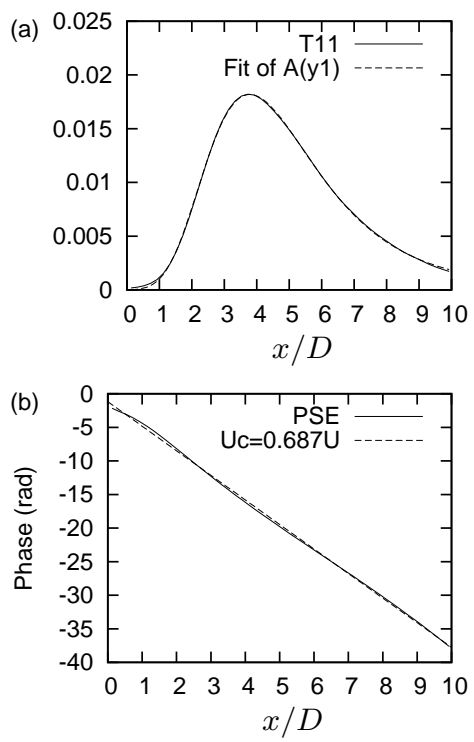

(c)
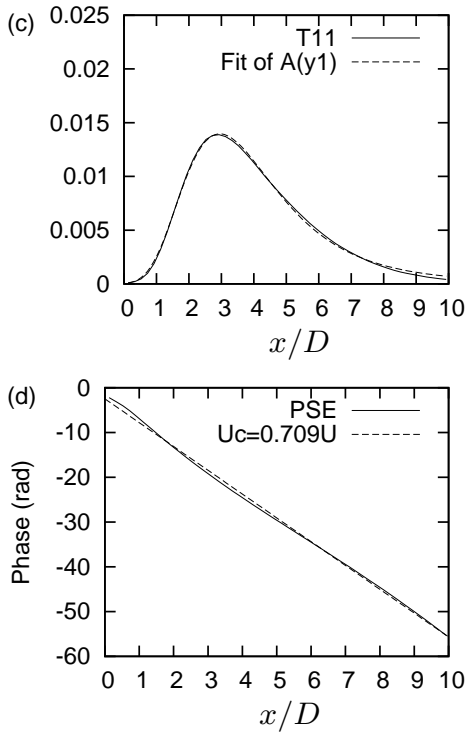

(e)
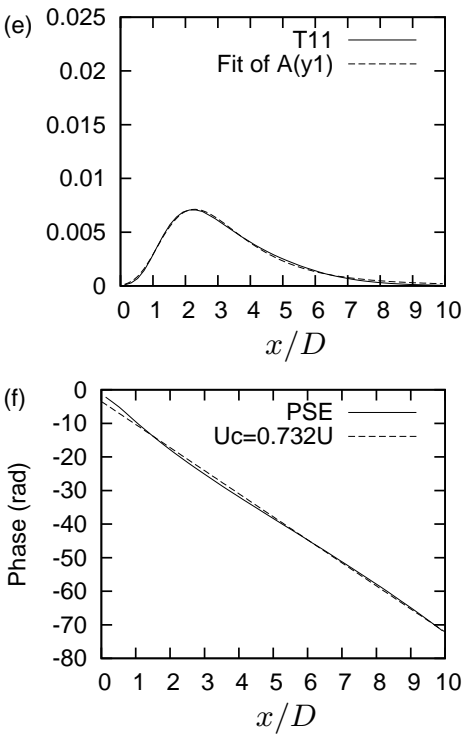

Figure 6. Fits of amplitudes and phases for the PSE modes of the $M_{j}=0.4$ jet and (a) and (b), $S t=0.4$; (c) and (d), $S t=0.6$; and (e) and (f), $S t=0.8$

Comparison of the sound radiation using equations (6) and (7) with the measured sound field for the axisymmetric mode is shown in figure 7 for the $M_{j}=0.4, M_{j}=0.5$ and $M_{j}=0.6$ jets. Note that the angle $\theta$ is measured here with respect to the aft direction, as opposed to figure 2 . As described before, the model uses the experimental mean velocity field and the PSE modes for the axial velocity, fitted with the experimental data for velocity fluctuations; hence, no information on the measured acoustic field is used for 

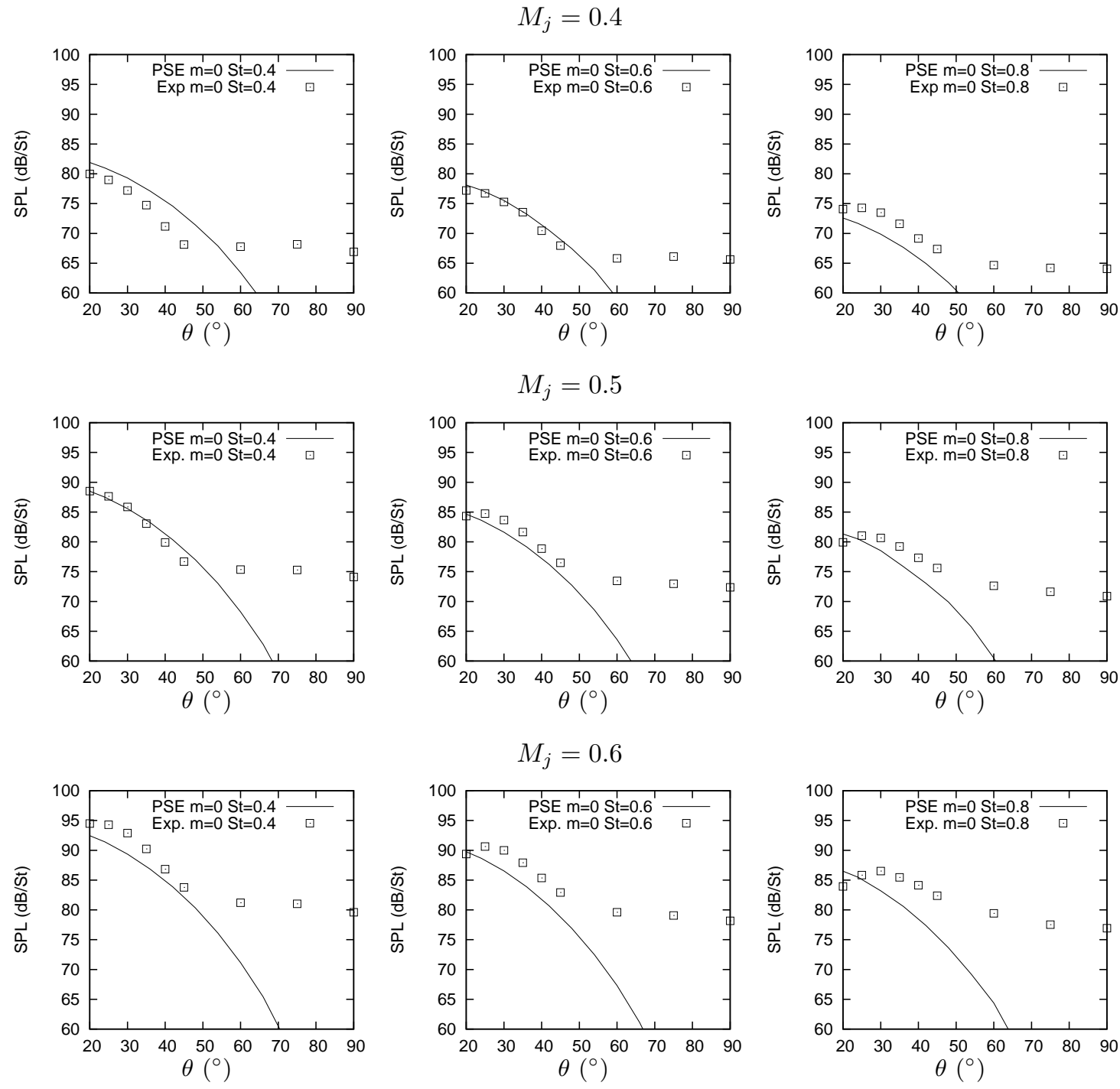

Figure 7. Comparison between sound fields predicted by projection of the linear PSE results and experiment.

calibration. There is good agreement (within $3 \mathrm{~dB}$ ) for the Strouhal number range of 0.4-0.9. The present model is able to predict both the absolute sound pressure level and the correct trends as the Mach number is increased. This supports the contention that instability waves are responsible for the sound radiation at low axial angles, even for these low Mach number jets. Comparison of the far field pressure levels for the different Mach numbers shows that the sound intensity increases with the Mach number. In the previous section, it was shown that the amplitude of the PSE modes increases with decreasing Mach numbers, remarking one again the strong influence of Mach number on the radiation efficiency of wavepackets: the instability waves modeled by PSE represent a non-compact source, spanning several jet diameters; thus, significant interference effects between the different axial stations are present inside such a source, and the increase of the Mach number, with the consequent reduction of the acoustic wavelength relative to the jet diameter, plays a major role in changing the interference pattern. This leads to higher acoustic intensities, even though the amplitudes of the instability waves are lower.

\section{Nonlinear PSE computations}

The linear results presented in the previous section give further evidence that the large-scale structures present in unforced turbulent jets can be satisfactorily modeled using wavepackets computed via linear stability theory ${ }^{12}$ or more sophisticated and ad hoc techniques like PSE. ${ }^{1,2}$ However, while the energy 
Table 1. Parameters defining the different frequency modes selections

\begin{tabular}{cccc}
\hline & $\Delta S t$ & $S t_{\max }$ & Total number of frequency modes \\
\hline \hline Case A & 0.1 & 0.5 & 6 \\
Case B & 0.1 & 0.8 & 9 \\
Case C & 0.05 & 0.8 & 17 \\
\hline
\end{tabular}

contained in the individual large-scale structures in unforced turbulent jets is very small compared with that of the mean flow or fine-grained turbulence, argument that has been used in the past to neglect the non-linear terms, ${ }^{9}$ it is not necessarily small enough to neglect the interactions between the different wavelengths and frequencies in the PSE.

In this section nonlinear PSE are applied to the same turbulent jets that were considered in §IIIA. In particular, the $M_{j}=0.9$ cold jet (SP7 according to Tanna's case matrix ${ }^{29}$ ) is revisited here. Following previous experience (see §IIIA), the POD-filtered experimental modes are used here in order to determine the initial conditions to be imposed in nonlinear PSE computations: first, a linear PSE computation is done for the mean flow at hand. The modal amplitudes are then scaled in order to obtain the best agreement (in the sense of least mean square error) with the first six microphone rings, spanning the first 4 diameters, where nonlinear interactions are assumed to be small. The modal amplitudes $A_{m n}\left(x=x_{0}\right)$ obtained in this manner are then imposed as initial conditions for the nonlinear computations. While this procedure permits determining appropriate initial values for the numerical integration, whether they represent adequately the true physics of turbulent jets requires further discussion. This aspect will be revisited in $§ I V B$.

\section{A. Effect of the frequency truncation}

As opposed to linear PSE computations in which the evolution of each mode is independent, nonlinearity couples the evolution of all the frequencies and azimuthal wavenumbers. The unavoidable truncation in the number of modes comprised in the computation alters the evolution of any individual mode. In the PSE formulation, two frequencies determine the choice of modes: the lowest frequency resolved (which we term here the fundamental frequency) and the truncation frequency. In the present investigation, the importance of the fundamental frequency lies in the fact that it determines the difference in frequency (from now on, $\Delta S t$ ) between two consecutive frequency modes, as well as the lowest frequency introduced in the computations. PSE equations are not adequate to deal with very low frequencies, and thus the choice of the fundamental frequency must done carefully. The highest or truncation frequency $S t_{\max }$ is also an arbitrary choice in the context of the turbulent jets, and determines which scales are treated as wavepackets and resolved, and which ones are considered fine-grained turbulence and modeled (equation (4)). Such an abrupt distinction does not exist in the true jet flow. The choice of $S t_{\max }$ must be done in order to recover consistent predictions for the most interesting range of frequencies $S t \approx 0.2-0.5$, in noting that the higher modes will not be accurate.

Three different cases will be considered in what follows, to investigate the effect of the choice of frequency modes. The parameters for each case are shown in table 1. Cases A and B have the same fundamental frequency $\Delta S t=0.1$, and different truncation frequencies. Case $\mathrm{C}$ has a fundamental frequency $\Delta S t=$ 0.05 , and a truncation frequency $S t_{\max }=0.8$. Nonlinear computations were conducted using these mode combinations, initialized in all cases with the same initial conditions. Figure 8 shows the pressure amplitudes extracted from the PSE results, at the location of the microphone array, for some selected modes in the computations. The figure also shows the first POD mode (circles) extracted from experimental data, as well as the results of linear PSE computations (thick red lines). First we investigate the effect of increasing the truncation frequency for a given frequency spacing (Cases A and B). The modal evolution is nearly identical for $S t \geq 0.3$, while minor differences are found for $S t=0.2$. For $S t=0.1, m=0$ a clear difference between cases A and B exists, but it is shown that its impact on the evolution of the higher frequency modes is negligible. In fact, comparing the nonlinear results with the linear ones, it can be concluded that the evolution of higher frequencies is, for the present unforced turbulent jet, nearly linear. Solid black lines correspond to the Case C, with $\Delta S t=0.05$. The evolution for most of the modes follows that described for cases A and B. However, a excessive peak value is attained for $S t=0.3, m=0$, as well as for $S t=0.6$ (not shown in the figure), in which the maximum pressure amplitude is almost doubled with respect to the linear case. 

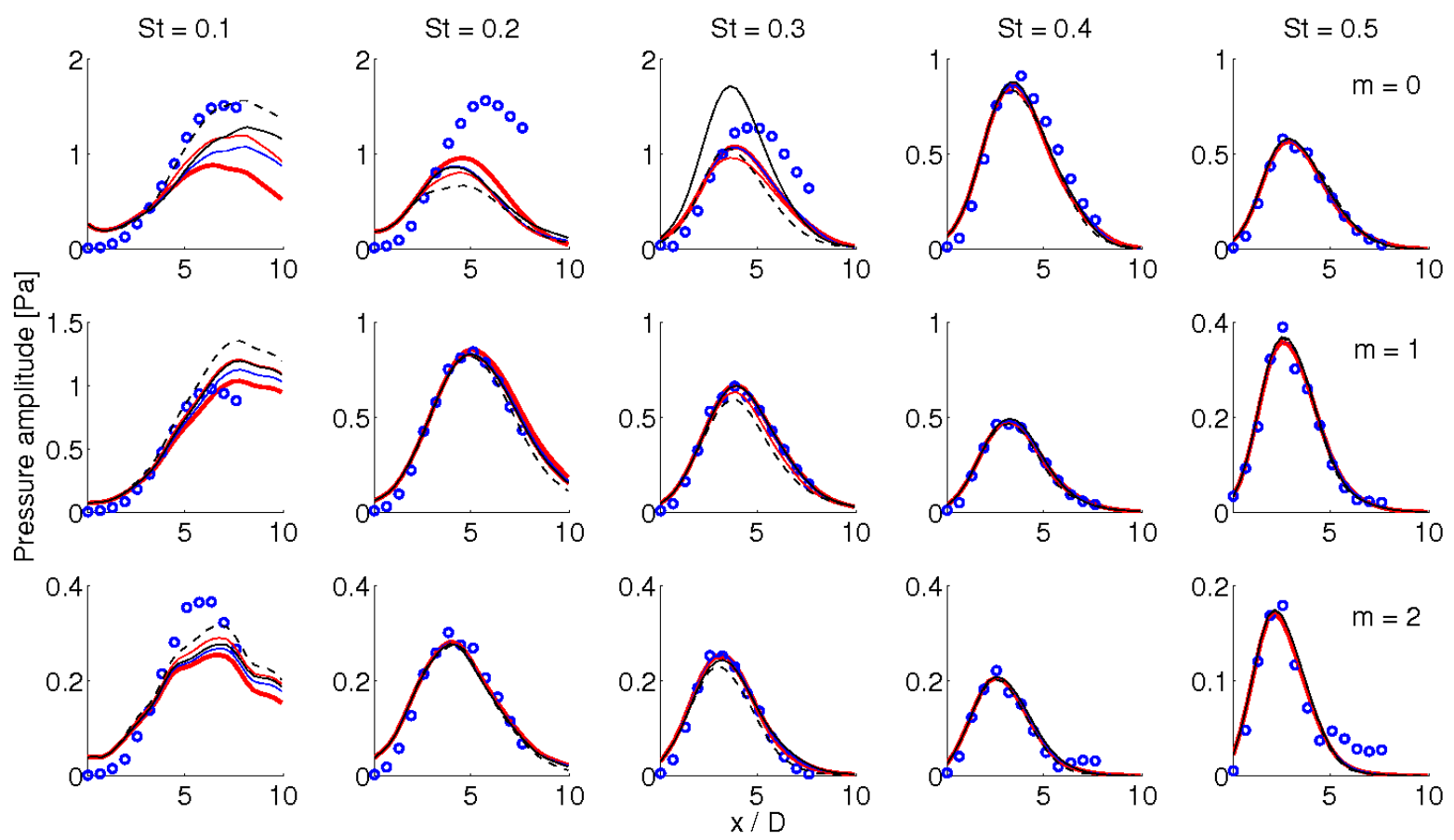

Figure 8. Pressure amplitude along the microphone array for the cold $M_{j}=0.9$ jet (SP7). Thick red lines correspond to linear PSE. Blue lines correspond to Case A, thin red lines to B, solid black lines to Case C and dashed black lines to Case C2. Blue circles correspond to the first POD mode.

One possible explanation for this change is that when the frequency spacing is reduced, initial amplitudes are assigned to the additional modes, thus increasing the total fluctuating energy, and permitting stronger nonlinear interactions. Another explanation is that the new lower frequency mode $S t=0.05$ corresponds to a frequency too low for the PSE approach to deliver consistent results, and consequently this mode can give rise to unphysical behaviors in other modes via nonlinear interaction. In order to investigate this last possibility, a new Case $\mathrm{C} 2$ was computed. Case $\mathrm{C} 2$ is identically the same as Case $\mathrm{C}$, but the initial amplitude of the $S t=0.05$ modes is neglected ${ }^{\mathrm{a}}$. The dashed black lines in figure 8 correspond to this last case, showing consistent predictions with those obtained for $\Delta S t=0.1$. This experience remarks the importance of an appropriate selection of the frequency modes.

\section{B. Random perturbation of the initial conditions}

In the previous sections, fixed modal amplitudes and phases were used to initialize the computations. These initial amplitudes were determined via a Power Spectral Density (PSD) estimation of the experimental time series, implicitly averaging the pressure amplitudes over a long lapse of time. In this sense, the initial amplitudes used provide a representation of the mean initial conditions observed in the experiments. However, the instantaneous pressure measured in the microphones shows considerable deviations from the PSD values along time, and their effect on the nonlinear computations should be taken into account.

There are several possibilities on how to deal with the initial conditions, in order to better reproduce the true physics of natural turbulent jets. The most straight forward approach is considering the initial conditions for the modes, and then the PSE computations, as deterministic. In this case, experimental pressure series are considered as one realization of the jet flow over a lapse of time. Instead of estimating a PSD, the time series are simply Fourier transformed to obtain the adequate amplitudes and phases for the nonlinear PSE to reproduce the realization. The nonlinear results obtained in this manner for each individual realization do not provide an adequate description of the true turbulent jet, but can be used to obtain bounds for the evolution of the wavepackets, and averaged to deliver a mean description of the flow.

\footnotetext{
${ }^{a}$ In practice, set equal to $10^{-6}$, few orders of magnitude lower than the amplitudes of the other modes.
} 

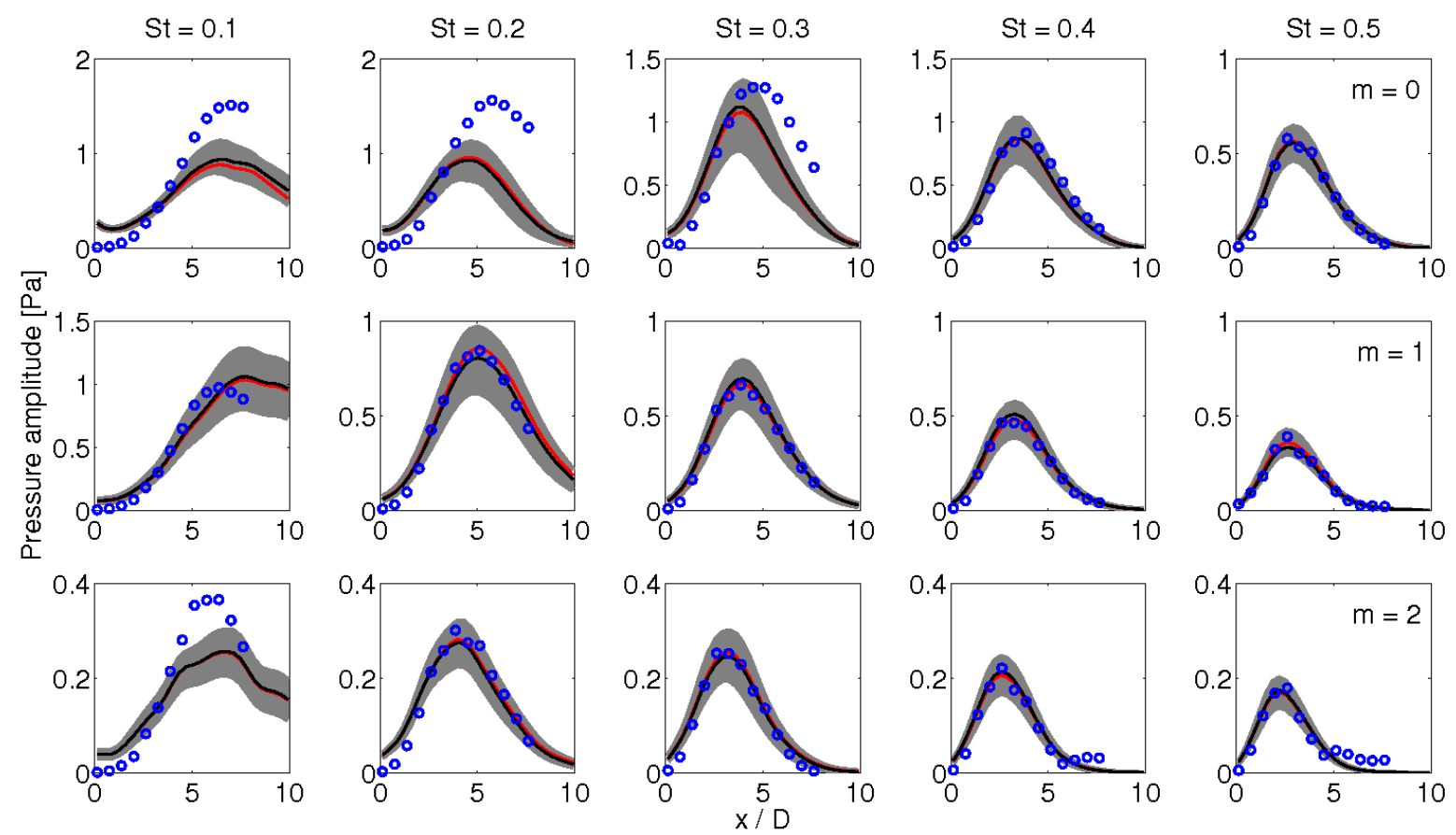

Figure 9. Pressure amplitude along the microphone array for the cold $M_{j}=0.9$ jet (SP7). Black line corresponds to the average and grey shaded region the bounds of 15 randomized nonlinear PSE computations. Red line corresponds to linear PSE. Blue circles correspond to the first POD mode.

The main shortcoming of this approach is the need for a relatively large number of realizations in order to provide unbiased results.

On the other hand, the evolution of the large-scale structures can be analyzed in a stochastic manner. Instead of introducing the (averaged) PSD estimations as initial conditions, the experimental data is processed to extract probability distribution functions (PDF) for the initial modal amplitudes and phases. Following methods from classical statistical mechanics, ${ }^{34}$ the Parabolized Equations should then be modified to serve as transport equations for the parameters defining the PDFs mentioned above. Additional complications are present in this approach, regarding the adequate PDFs to be used, or the impact of nonlinear interactions on the behavior of the model equations.

An intermediate approach is used here. As for the previous sections, the experimental time series for pressure are used to build a PSD estimation, and a 'basic' initial amplitude $A_{m n}^{*}\left(x=x_{0}\right)$ is thus determined for every mode. The initial condition is then randomized both in amplitude and phase:

$$
A_{m n}\left(x=x_{0}\right)=A_{m n}^{*}\left(x=x_{0}\right) \cdot \sigma \cdot \mathrm{e}^{\mathrm{i} \mu},
$$

where $\sigma$ is a random number in the range $\sigma=[0.8-1.2]$ following a normal probability distribution, and $\mu$ is an arbitrary phase with a constant probability distribution, and taking values $\mu=[0,2 \pi]$. The values $\sigma$ and $\mu$ are determined independently for each mode in every computation.

Figure 9 shows averaged pressure amplitude distributions (black lines) for some selected modes delivered by 15 independent nonlinear runs with randomly perturbed initial amplitudes and phases. The shaded grey regions bounds the evolution of the modes in the nonlinear computations. For comparison, the results of the linear computation and the first POD mode extracted from microphone data, used to determine the amplitudes $A_{m n}^{*}$ are plotted overlaid. A fixed number of frequency $S t=[0,(0.1), 0.8]$ and azimuthal $m=[0,1,2]$ modes was used in all computations. A remarkable effect of the initial phases and amplitudes appear at the lower frequencies, especially for $S t=0.1, m=0$. However, as the frequency is increased the nonlinear effects are less evident. The dispersion in the pressure amplitudes for $S t \geq 0.3$ is attributed to the randomized initial amplitudes: due to the nearly exponential growth of the modes, very small deviations in the initial conditions at the nozzle result into $O(1)$ differences in the peak values. These differences are not 
caused by nonlinearity, as the amplitude envelope is nearly the same for all the random cases.

\section{Investigation of difference-mode nonlinear interactions}

In the previous sections it was shown that wavepackets recovered using PSE are the source of the superdirective noise radiation to the far field, at least for frequencies higher than $S t=0.3$. It was also shown that for this range of frequencies, the nonlinear interactions were negligible in the evolution of the PSE modes. Experimental observations ${ }^{35,36}$ indicate the presence of a broadband spectral peak near $S t=0.2$ at small angles with the nozzle axis. The possibility that this low frequency noise is generated by a nonlinear process has been investigated experimentally ${ }^{4}$ and numerically; ${ }^{5}$ their conclusion is that the nonlinear interaction of two forced modes excites the difference frequency mode in a manner such that the radiated noise to the far field is dominant. They concluded that forcing $S t=0.3$ and $S t=0.5$ modes produce an optimal radiation for the $S t=0.2$ mode, which is higher than the one obtained by forcing the $S t=0.2$ frequency directly.

It is unclear whether such a mechanism is at play in natural, turbulent jets. Two fundamental aspects in the simulations of Suponitsky et al. differ from the natural jets of study in the previous sections. First, a laminar jet flow at Reynolds number 3600 was used, two orders of magnitude than the $O\left(10^{5}\right)$ jets considered in the experiments of Suzuki and Colonius. ${ }^{12}$ Second, only the two forced modes were intialized with a finite amplitude, avoiding any mode-to-mode interaction other than the one originating the frequency-difference mode, and the ones responsible for higher frequency modes. In a natural turbulent flow a broad spectrum of fluctuations exists, so the initial amplitudes given to the modes will be of the same order of magnitude for a large number of modes, and the resulting nonlinear interactions will be more complex, possibly reducing the efficiency in the excitation of the difference mode. To investigate this possibility, we use nonlinear PSE with two different sets of initial conditions that attempt to account for this difference between forced and natural jets. The first setup will be referred to as forced, and tries to mimic the computations of Suponitsky et $a .^{5}$ In this case, finite initial amplitudes will be assigned to $S t=0.3$ and $S t=0.5$ modes only, so the generation of other modes will be caused exclusively by nonlinear interactions. The second setup will be termed natural. In this case, the initial amplitudes $A_{m n}^{*}\left(x=x_{0}\right)$ obtained from the experimental data are assigned to all the modes introduced in the computations, as was done in the previous sections. Then, the amplitude of the $S t=0.3$ and $S t=0.5$ modes is increased over the experimental value, in order to simulate the effect of forcing on a realistic natural jet.

Table 2 shows the different cases considered, compared with cases extracted from Suponitsky et al. ${ }^{5}$ Some differences exist between the computations in the reference and the present ones. The first difference is the mean flow. Instead of a analytical laminar base flow at Reynolds number $3600,{ }^{5}$ the mean turbulent flow measured experimentally corresponding to the cold $M_{j}=0.9$ jet is used here, for which the Reynolds number is $O\left(10^{5}\right)$. The second difference is the choice of the forced modes. In the present computations the forced modes are $S t=0.3$ and $S t=0.5$, and the difference mode to be excited is $S t=0.2$. In the reference, the forced modes are $S t \approx 0.315$ and $S t \approx 0.487$ and the difference mode $S t \approx 0.179$. The small differences on these values are expected not to have a strong impact on the nonlinear excitation process.

Two parameters are introduced here in order to illustrate the effect of nonlinear interactions on the total fluctuating flow and on the frequency-difference mode. The first parameter, denoted as $\left(p_{r m s}\right)_{m a x} / p_{0, r m s}$ in table 2, measures the spatial amplification of the fluctuating pressure for all modes, as the ratio between the r.m.s. pressure magnitude at the nozzle lip $\left(p_{0, r m s}\left(x=x_{0}, r=0.5 D\right)\right)$, and the maximum r.m.s. pressure in the computational domain, $\left(p_{r m s}\right)_{\max }$. The evolution of this ratio with increasing forcing amplitudes is an indication of the level of nonlinearity present in the evolution of disturbances. In a completely linear evolution, this parameter would be constant. In nonlinear computations, as the initial modal amplitudes become higher, nonlinear interactions will saturate the disturbance growth, and the ratio will decrease. This behavior is shown in the forth column of table 2, for the cases extracted from Suponitsky et al. ${ }^{5}$ and those corresponding to the present forced computations. Both the evolution with increasing initial amplitudes and the absolute values of the parameter are very similar for these two cases. While the parameter values are reduced with increasing initial amplitudes, the actual reduction for the current range of amplitudes is very small. Consequently, the nonlinear interactions are concluded to have a limited effect on the global flow dynamics of the forced jets, at the forcing amplitudes considered, even though they will be able to excite the difference mode in a noticeable manner, as described later.

In the case of natural jets, the ratio takes lower values, that tend to the ones recovered in the forced cases as the initial amplitude is increased for $S t=0.3$ and $S t=0.5$ (last rows in table 2). This can be explained in the following manner: as was shown in the previous sections, the evolution of these frequencies is nearly 
Table 2. Inflow parameters and amplification of the total fluctuating pressure and mode $S t=0.2$.

\begin{tabular}{|c|c|c|c|}
\hline & $A_{3,0}$ & $\left(p_{r m s}\right)_{\max } / p_{0, r m s}$ & $\left(p_{S t=0.2}\right)_{\max } / p_{0, S t=0.2}$ \\
\hline \multirow{3}{*}{$\begin{array}{c}\text { Results from } \\
\text { reference } 5\end{array}$} & $1 \times 10^{-5}$ & $1.736 \times 10^{2}$ & - \\
\hline & $1 \times 10^{-4}$ & $1.736 \times 10^{2}$ & - \\
\hline & $5 \times 10^{-4}$ & $1.622 \times 10^{2}$ & - \\
\hline \multirow[t]{3}{*}{ Present forced cases } & $1 \times 10^{-5}$ & $1.109 \times 10^{2}$ & $1.349 \times 10^{2}$ \\
\hline & $1 \times 10^{-4}$ & $1.067 \times 10^{2}$ & $1.062 \times 10^{3}$ \\
\hline & $5 \times 10^{-4}$ & - & - \\
\hline \multirow[t]{3}{*}{ Present natural cases } & $3.593 \times 10^{-5} \quad 1.470 \times 10^{-5}$ & $4.861 \times 10^{1}$ & $7.733 \times 10^{0}$ \\
\hline & $1 \times 10^{-4}$ & $7.414 \times 10^{1}$ & $8.509 \times 10^{0}$ \\
\hline & $5 \times 10^{-4}$ & $8.916 \times 10^{1}$ & $1.496 \times 10^{1}$ \\
\hline
\end{tabular}

linear; increasing the initial amplitude of the two modes gives them a higher relative importance over the other modes in the total pressure field. Consequently, the total pressure field tends to behave in a similar manner to the combination of the two excited modes alone.

The second parameter, shown in the last column of table 2, measures the relative excitation of the $S t=0.2$ difference mode. This parameter is the ratio between the maximum pressure fluctuation corresponding to this mode in the computational domain $\left(\left(p_{S t=0.2}\right)_{\max }\right)$, and the pressure amplitude at the nozzle lip, $p_{0, S t=0.2}$. Suponitsky et al. ${ }^{5}$ do not provide enough information to compute this ratio. For the forced cases, this parameter attains large values and grow proportional to the initial amplitudes of the forced modes, illustrating that its growth is effectively dominated by their nonlinear interaction. However, the peak amplitudes reached by the difference mode are much lower than those of the forced $(S t=0.3$ and 0.5) modes: in the cases presented, the generation of a difference-frequency mode by means of forcing two modes with higher frequency is less efficient than forcing directly the mode. Unfortunately, nonlinear PSE computations failed to converge for the present mean flow when higher initial amplitudes were imposed to the forced modes, avoiding comparisons with the higher nonlinear levels of Suponitsky et al. ${ }^{5}$ In the case of the natural jets, the nonlinear excitation of the difference mode is drastically reduced when compared to the forced case. This can be explained by the presence of a multitude of finite-amplitude modes in the natural case, that reduces the strength of mode-to-mode nonlinear interactions responsible for the excitation of the difference mode in the much "cleaner" forced jet cases.

\section{Conclusions}

This paper uses nonlinear Parabolized Stability Equations in order to model the large-scale turbulent structures responsible for the main part of far field noise radiated in the aft direction in natural jets. In previous research using linear PSE ${ }^{2}$ it was found that a very good agreement exists between the amplitude envelope and phase of the predicted wavepackets with experimental measurements on a phased microphone array, placed just outside of the jet shear layer. Matching the wavepacket amplitudes with those in this array, and using a Kirchhoff projection method, it was shown that the noise radiated to the far field by the wavepacket is in good agreement with experiments. ${ }^{18}$ In this paper, a similar approach was applied to a separate set of experimental data. ${ }^{3}$ Linear PSE was employed to recover wavepackets corresponding to low Mach number $\left(M_{j}=0.4\right.$ to 0.6$)$ natural jets. Hot-wire measurements on the jet centerline were used in order to match the wavepacket amplitudes: very good agreement in the amplitude envelopes was found between theory and experiments for $S t \geq 0.3$ frequencies, in a region extending up to the end of the potential core. Subsequently, the computed wavepackets were used to build a model noise source for the acoustic analogy method. Again, good comparisons were obtained in the shape and amplitudes of the directivity curves for the far field noise. The fact that good predictions of far field noise were obtained here using only experimental velocity data for determining the mode amplitudes provides further evidence in that instability waves are responsible for the sound radiation at low axial angles, even for these low Mach number jets.

The second part of the paper focuses on the application of nonlinear PSE in order to improve the 
wavepacket description. Two main conclusions can be drawn from the nonlinear results presented. The first one is related to the evolution of low frequency modes and how nonlinearity can extend their influence into the higher frequency modes. The applicability of the PSE approach to low frequency modes can be questioned when the associated wavelength is long enough so that it is comparable to the scale of variation of the base flow. This is the case of the $S t \leq 0.1$ modes, for which the wavelength is comparable to the potential core, and explains why the evolution of these frequencies is so sensitive to the initial amplitudes and phases, truncation frequency and frequency spacing. The second main conclusion is that the evolution of the modes with frequency $S t \geq 0.3$ is nearly linear in most cases, and consequently is robust against changes in the initial conditions and the choice of the frequency modes.

Finally, a model nonlinear interaction mechanism ${ }^{4,5}$ is investigated in the scope of nonlinear PSE. This model suggests that the peak noise in the far field corresponds to a $S t=0.2$ mode which is most efficiently generated by the nonlinear interaction of two modes with a higher frequency $(S t=0.3$ and $S t=0.5)$. A series of computations were conducted mimicking the conditions of a "clean" forced jet, and a natural turbulent jet, for which these two modes are forced. It has been shown that in natural turbulent jets, the existence of a very complex modal system drastically reduces the efficiency of the nonlinear mechanism of excitation of the difference mode, when compared to much "cleaner" low Reynolds number jets.

This work was supported in part by NAVAIR through an SBIR contract to TTC Technologies, Inc. The technical monitor was Dr. John Spyropoulos. André Cavalieri is founded by CNPq, Brazil. The authors would like to thank Dr. Kristjan Gudmundsson, and Drs. Robert Schlinker and Ramons Reba of United Technologies Research Center for their input on this work.

\section{References}

${ }^{1}$ K. Gudmundsson, Instability wave models of turbulent jets from round and serrated nozzles, Ph.D. thesis, California Institute of Technology (2010).

${ }^{2}$ K. Gudmundsson, T. Colonius, Instability wave models for the near-field fluctuations of turbulent jets, J. Fluid Mech, submitted.

${ }^{3}$ A. V. G. Cavalieri, P. Jordan, Y. Gervais, T. Colonius, Axisymmetric superdirectivity in subsonic jets, in: 17th AIAA/CEAS Aeroacoustics Conference, 6-8 June Portland, OR, 2011.

${ }^{4}$ D. Ronneberger, U. Ackermann, Experiments on sound radiation du to non-linear interaction of instability waves in a turbulent jet, J. Sound Vib. 62 (1) (1979) 121-129.

${ }^{5}$ V. Suponitsky, N. Sandham, C. Morfey, Linear and nonlinear mechanisms of sound radiation by instability waves in subsonic jets, J. Fluid Mech. 658 (2010) 509-538.

${ }^{6}$ M. Lighthill, On sound generated aerodynamically. I. General theory, Proc. R. Soc. Lond. A. 211 (1107) (1952) 564-587.

${ }^{7}$ E. Mollo-Christensen, Jet noise and shear flow instability seen from an experimenter's viewpoint, J. Applied Mech. 34 (1967) $1-7$.

${ }^{8}$ J. T. C. Liu, Developing large-scale wavelike eddies and the near jet noise field, J. Fluid Mech. 62 (1974) 437-464.

${ }^{9}$ R. Mankbadi, J. T. C. Liu, A study of the interactions between large-scale coherent structures and fine-grained turbulence in a round jet, Proc. Roy. Soc. London 1443 (1981) 541-602.

${ }^{10}$ P. Morris, M. Giridharan, G. Lilley, On the turbulent mixing of compressible free shear layers, Proc. R. Soc. Lond. A. 431 (1990) 219-243.

${ }^{11}$ C. Tam, P. Morris, Tone excited jets - Part V: A theoretical model and comparison with experiments, J. Sound Vib. 102 (1985) 119-151.

${ }^{12} \mathrm{~T}$. Suzuki, T. Colonius, Instability waves in a subsonic round jet detected using a near-field phased microphone array, J. Fluid Mech. 565 (2006) 197-226.

${ }^{13}$ K. Gudmundsson, T. Colonius, Parabolized stability equation models for turbulent jets and their radiated sound, AIAA Paper 2009-3380.

${ }^{14}$ N. Sandham, A. Salgado, Nonlinear interaction model of subsonic jet noise, Phil. Trans. Roy. Soc. A 366 (2008) 2745-2760.

${ }^{15}$ M. R. Malik, C. L. Chang, Nonparallel and nonlinear stability of supersonic jet flow, Comp. Fluids 29 (2000) 327-365.

${ }^{16}$ J. Nichols, S. Lele, P. Moin, Global mode decomposition of supersonic jet noise, in: Center for Turbulence Research Annual Research Briefs, Stanford, CA, 2009.

${ }^{17}$ R. Reba, S. Narayanan, T. Colonius, Wave-packet models for large-scale mixing noise, Int. J. Aeroacoustics 9 (4 \& 5) (2010) 533-558.

${ }^{18}$ T. Colonius, A. Samanta, K. Gudmundsson, Parabolized stability equation models of large-scale jet mixing noise, Procedia Engineering 6 (2010) 64-73.

${ }^{19}$ S. C. Crow, Acoustic gain of a turbulent jet, Vol. 6, Phys. Soc. Meeting, Univ. Colorado, Boulder. paper IE, 1972.

${ }^{20}$ D. G. Crighton, Basic principles of aerodynamic noise generation, Progress in Aerospace Sciences 16 (1) (1975) 31-96.

${ }^{21}$ F. P. Bertolotti, T. Herbert, Analysis of the linear stability of compressible boundary layers using the PSE, Theoret. Comput. Fluid Dyn. 3 (1991) 117-124. 
${ }^{22}$ F. P. Bertolotti, T. Herbert, P. Spalart, Linear and nonlinear stability of the blasius boundary layer, J. Fluid Mech. 242 (1992) 441-474.

${ }^{23}$ T. Herbert, Parabolized stability equations, Annu. Rev. Fuid Mech. 29 (1997) 245-283.

${ }^{24}$ K. Thompson, Time dependent boundary conditions for hyperbolic systems, J. Comp. Phys. 68 (1987) 1-24.

${ }^{25}$ F. Li, M. R. Malik, Spectral analysis of parabolized stability equations, Comp. Fluids 26 (3) (1997) $279-297$.

${ }^{26}$ M. J. Day, N. N. Mansour, W. C. Reynolds, Nonlinear stability and structure of compressible reacting mixing layers, J. Fluid Mech. 446 (2001) 375-408.

${ }^{27}$ B. Noack, K. Afanasiev, M. Morzynski, G. Tadmor, F. Thiele, A hierarchy of low-dimensional models for the transient and post-transient cyclinder wake, J. of Fluid Mech. 497 (2003) 335-363.

${ }^{28}$ H. Schlichting, Boundary layer theory, 7th Edition, McGraw-Hill, 1979.

${ }^{29}$ H. K. Tanna, An experimental study of jet noise. part I: Turbulent jet noise, J. Sound Vib. 50 (3) (1977) $405-428$.

${ }^{30} \mathrm{~J}$. Bridges, M. Wernet, Measurements of the aeroacoustic sound source in hot jets, AIAA Paper 2003-3130.

${ }^{31}$ A. V. G. Cavalieri, D. Rodríguez, P. Jordan, T. Colonius, Y. Gervais, Inlet conditions and wave-packets in subsonic jet noise, in: Turbulence and Shear Flow Phenomena, Ottawa, Canada. July 28-31, 2011.

${ }^{32}$ A. V. G. Cavalieri, P. Jordan, A. Agarwal, Y. Gervais, Jittering wave-packet models for subsonic jet noise, to appear in Journal of Sound and Vibration.doi:10.1016/j.jsv.2011.04.007.

${ }^{33}$ M. Køenig, A. V. G. Cavalieri, P. Jordan, J. Delville, Y. Gervais, D. Papamoschou, M. Samimy, S. K. Lele, Farfield pre-filterering and source-imaging for the study of jet noise, in: 16th AIAA/CEAS Aeroacoustics Conference and Exhibit, Stockholm, Sweden, 2010.

${ }^{34}$ S. Heinz, Statistical Mechanics of Turbulent Flows, Springer, 2003.

${ }^{35}$ J. L. Stromberg, D. K. McLaughlin, T. R. Troutt, Flow field and acoustic properties of a mach number 0.9 jet at low reynolds number, J. Fluid Mech. 72 (1980) 159-176.

${ }^{36}$ K. Viswanathan, Aeroacustics of hot jets, J. Fluid Mech. 516 (2004) 39-82. 